\title{
Entrenamiento psicológico de los especialistas en los deportes de equipo. Una experiencia en voleibol
}

\section{Psychological training of specialists in team sports. An experience in volleyball}

Fecha de recepción: 15/10/2016

Fecha de aceptación: 25/10/2016

\author{
Joaquín Díaz Rodríguez \\ Psicólogo. Máster en Psicología del Deporte \\ Comité Nacional Entrenadores RFEVB \\ Federación Española de Psicología del Deporte FEPD
}

España

\section{resumen/abstract:}

En este trabajo abordaremos el trabajo psicológico para implantar y mejorar las habilidades psicológicas que requieren los líberos, uno de los especialistas en voleibol. Presentamos la forma de trabajo de intervención y asesoramiento psicológico para el entrenamiento de la defensa de segunda línea en voleibol y para implementar y mejorar las habilidades psicológicas de los especialistas en ese trabajo defensivo. La propuesta de trabajo puede ser un modelo de intervención psicológica para complementar las tareas deportivas que deben realizar aquellos jugadores que ocupan puestos de especial relevancia e importancia en el juego colectivo. Se aporta a los entrenadores formas de actuación, tanto de trabajo personalizado, como integrado en la sesiones de entrenamiento, es decir: entrenamientos psicológicamente orientados, que es uno de los retos que nos debemos plantear los psicólogos especializados en deporte.

In this work we address the psychological work to implement and improve the psychological skills needed by liberos, one of the specialists in volleyball. We present the form of intervention work and psychological counseling for defense training in volleyball second line to implement and improve the psychological skills of specialists in the defensive work. The proposed work can be a model of psychological work to complement the sporting tasks which have to perform those players who hold positions of particular relevance and importance in the collective game. It provides coaches with ways of acting, both in their personal work and in the ones integrated in their training sessions, such as psychologically oriented training, which is one of the challenges that sport psychology specialist must raise.

\section{palabras clave/keywords:}

Entrenamiento psicológico, especialistas, equipos, voleibol.

Psychological training, Specialists, Teams, Volleyball.

\section{Introducción}

Muchos trabajos postulan la importancia del trabajo psicológico y describen programas de entrenamiento psicológico o intervenciones con los deportistas (Buceta, 1992; Cruz, 1992, Escudero López, 1992; Pérez, Marí, y Font, 1993; Pulgarín Medina, 1996; Blanco \& Buceta, 1999; Nieto y Olmedilla, 2001; Eraña de Castro, 2004; González y Garcés de los Fayos, 2009; Díaz 2010). Existen, también, diferentes trabajos sobre entrenamientos psicológicos de jugadores especialista: intervención psicológica en porteras de balonmano (Antúnez, 
Ureña, Velandrino y García Parra, 2004), y un estudio piloto sobre el mismo especialista masculino (Medina, Parra, Iturriaga, Lara, y Estero, 2010), tareas de porteros de futbol profesional (De la Vega, Barquín y Valle, 2010), percepción del éxito en porteros de waterpolo (Iturriaga, Marcos, y Barquín, 2015), porteras de hockey hierba (Sánchez-Alarcos y Díaz, 2015), porteros de balonmano ( Sá, Gomes, Saavedra y Fernández, 2015), colocadores de voleibol (Noce y Samulsky, 2002). En este trabajo reflexionaremos sobre el trabajo psicológico para implantar y mejorar las habilidades psicológicas que se requieren para realizar las tareas propias del libero en voleibol como un especialista en tareas de recepción y defensa de segunda línea. Sin embargo, se harán referencias continuas como modelo de intervención psicológica para otros especialistas de otras modalidades deportivas

El proceso de entrenamientos específicos, personalizados y por tareas concretas y exclusivas para puestos especiales es, quizás, una de las particularidades del entrenamiento moderno en los deportes de equipo. En un gran número de modalidades de deportes de equipo existen uno o más puestos ocupados por especialistas que requieren poseer habilidades y capacidades especiales, tanto técnicas y tácticas como físicas y, por supuesto, psicológicas.

Los entrenamientos para las tareas específicas de líneas tácticas dentro de los equipos es otra tendencia del entrenamiento actual. Los defensas y los centrocampistas en fútbol (Olmedilla, Ortega, Ortín y Andreu, 2010), los lanzadores en balonmano (Martínez, Bonet y Encinas, 1998), los escoltas y pivots en baloncesto, (Jiménez, Sáenz-López, Ibáñez y Lorenzo. 2012) o los centrales y los receptores en voleibol (Bossio, Raimundi y Correa 2012) son algunos modelos de tareas tácticas específicas que requerirían entrenamientos psicológicos específicos.

Muchos equipos cuentan con entrenadores determinados que individualizan sus tareas con jugadores especialistas o por trabajos para puestos concretos. Los más característicos los porteros de fútbol y balonmano, los colocadores y líberos en voleibol, los bases de baloncesto.

La colaboración entrenador-psicólogo es la idónea para mejorar y aumentar el rendimiento deportivo de estos especialistas y de esas especificidades técnico-tácticas en los que los componentes mentales ocupan una parte importante del entrenamiento. Si los entrenadores inciden sobre la importancia del dominio de habilidades técnicas y tácticas concretas para los puestos específicos, el psicólogo del deporte tendrá como objeto recabar cuales son las habilidades psicológicas más necesarias para su optimo desempeño y en como implantarlas y mejorarlas mediante su entrenamiento.

El presente trabajo, por tanto, pretende ser una nueva aportación para los entrenadores de voleibol y para los psicólogos del deporte que aspiren a lograr una mayor especialización en este deporte. También pretende ser una guía para aquellos jugadores que realizan la funciones de especialistas y que encuentren en ella recursos, formas, estrategias, códigos y tareas mentales que les permitan realizar mejor y más saludablemente su especialidad. Además puede ser una guía para los trabajos psicológicos con otros jugadores especialistas.

La mayoría de los autores citados anteriormente, coinciden en que las aportaciones de la psicología del deporte al entrenamiento mental de estos especialistas optimizarán su traba- 
jo técnico-táctico, perfeccionarán sus habilidades psicológicas, potenciarán su fortaleza y control mental, y mejorarán la interactuación con el resto de sus compañeros en la dinámica general del juego de equipo.

Es cierto que en los últimos tiempos estamos teniendo la oportunidad de vivir como el mundo del deporte se está transformando y son cada vez más los entrenadores que aceptan la figura del psicólogo integrándolo en los staff técnicos de sus equipos. Trabajos como éste, siempre desde la prudente modestia, pretenden estrechar más esta relación aportando nuevas formas de trabajo integrado.

Somos muchos los entrenadores y psicólogos del deporte que estamos totalmente convencidos de que los jugadores en general, y los especialistas en particular, funcionarán y desarrollarán mejor sus técnicas y habilidades técnico-tácticas si progresan y amplían sus habilidades mentales. También progresarán más todos aquellos jugadores que reciban entrenamientos psicológicos apropiados a sus tareas que los que no los reciben.

Sin duda, actuarán mejor aquellos deportistas que, por ejemplo, tengan un buen control de la atención o un buen nivel de confianza y autoestima que aquellos que no lo tengan. Funcionarán mejor los equipos que mantengan un alto grado de colaboración y cooperación que aquellos que no lo hagan. Obtendrán mejores rendimiento aquellos equipos que cuenten con mejores especialistas y mejor formados.

En un primer momento, y como metodológica para el trabajo psicológico con los especialistas, es necesario conocer cuales son las destrezas físicas, técnicas y tácticas que requieren para desarrollar su cometido específico en el equipo.

En un segundo momento para el trabajo psicológico es imprescindible identificar cuales son las habilidades mentales que estos especialistas demandan para ejecutar esas destrezas o habilidades físicas y técnico-tácticas y así poder planificar programas de entrenamiento integrados. Según Mahl y Vasconcelos, (2007) son éstas habilidades mentales las que más contribuyen al logro de los niveles máximos de rendimiento.

Esta estrategia y dinámica de entrenamientos, en las que los aspectos mentales están inmersos, son ya aceptadas por una significativa mayoría de los entrenadores y cuadros técnicos. La aportación de los psicólogos del deporte a este modelo es esforzarse por aportar a la mejora y optimización de los entrenamientos los contenidos de la preparación psicológica y del entrenamiento mental.

A esta optimización y mejora de los rendimientos deportivos de los especialistas y del resto de jugadores, que es un objetivo fundamental de la psicología del deporte, los psicólogos del deporte deben procurar, como otro importante objetivo, el bienestar psicológico de estos especialistas, ya que, en muchas ocasiones, están sometidos a una mayor exigencia mental y a una mayor presión psicológica que el resto de compañeros, (Noce y Samulski, 2002).

Otra de las tareas que tienen los psicólogos del deporte dentro de los cuadros técnicos de los equipos es procurar herramientas de trabajo psicológico a los entrenadores para el trabajo conjunto con el resto de técnicos. 


\section{Características del libero como especialista en el voleibol}

Tareas técnico-tácticas y estratégicas encomendadas a los especialistas en los equipos y demandas psicológicas.

Para acometer una adecuada preparación psicológica del jugador especialista, en nuestro caso el líbero, es preciso conocer adecuadamente sus funciones en el entramado del equipo y además estar al tanto de las limitaciones reglamentarias de sus tareas, que en los líberos son características. Como ocurre con otros especialistas, por ejemplo los porteros de fútbol, waterpolo o balonmano, el líbero es un jugador de equipo que no puede realizar algunas acciones que para el resto de sus compañeros son obligatorias por reglamentarias, y a la inversa. Es necesario, también, estar al corriente de las características físicas, técnicas y tácticas que deben poseer, adquirir y mejorar los especialista. Por último, debemos conocer detalladamente cuáles son las exigencias psicológicas y mentales que requiere el puesto y sobre las que debemos intervenir para que realice sus tareas y funciones de especialista del equipo de manera óptima.

En el modelo de trabajo propuesto destacamos como las acciones tácticas propias del líbero las siguientes:

- Recepción del saque

- Defensa del ataque

- Defensa de apoyos (cobertura) del ataque propio

Como limitaciones reglamentarias, los líberos tienen las siguientes:

- No puede sacar.

- No puede atacar ningún balón que se encuentre por encima del borde superior de la red desde cualquier zona del campo.

- No puede bloquear.

- No puede participar en un bloqueo colectivo, ni siquiera realizar una tentativa de bloqueo.

- Aunque puede colocar con toque de dedos desde la zona de ataque, en caso de realizarlo, el atacante no puede completar ese ataque sobre dicho pase.

- En la zona de ataque, en caso de ser necesario un pase para ser atacado, el libero puede realizar apoyarse en el pase de antebrazos o con cualquier parte del cuerpo de la cintura hacia abajo.

Nivel técnico. Por regla general todos los especialistas de equipo deben poseer un excelente nivel técnico; dominar las técnicas generales, más aquellas que le son propias. Es el caso de los líberos, de los bases y pivots de baloncesto, los colocadores en voleibol o los quarterback en rugby. 
El nivel técnico del líbero debe ser muy exigente tanto para las tareas de defensa del saque (recepción) como para la defensa de segunda línea. De igual modo, debe poseer un buen segundo pase tanto de antebrazos como de dedos. Debe, por tanto, poseer las habilidades técnicas de un buen colocador, de un excelente receptor y de un defensor motivado. Los gestos técnicos que el líbero no puede realizar son: saque, bloqueo y remate, que son precisamente los gestos y acciones técnicas que aportan los puntos al equipo. Tiene permitido el pase de colocación desde la zona de ataque de tres metros, pero no puede ser rematado por delante de la línea de ataque. Se le exigen colocaciones desde posiciones y situaciones comprometidas. La sensación y visión de campo son fundamentales en los líberos excelentes. Los entrenamientos técnicos del líbero, por tanto, deben ser diferentes y específicos. Cada vez encontramos más equipos en los que aparecen entrenadores o ayudantes propios de los especialistas, que trabajan gran parte de las sesiones de entrenamiento aparte del grupo. Incluso en los partidos cada vez observamos con más frecuencia que los especialistas realizan un calentamiento técnico distinto al del resto de jugadores. Este aspecto es muy visible en los porteros de futbol y balonmano.

Nivel Táctico. Otro aspecto fundamental para el adecuado entrenamiento psicológico de los especialistas es conocer en profundidad las demandas tácticas que tiene encomendadas. Más arriba ya hemos comentado sus limitaciones tácticas reglamentarias

Hay especialistas como los bases en baloncesto y como los colocadores en voleibol en que prácticamente todo el entramado táctico del equipo y de los partidos pasa por sus manos y su cerebro.

El líbero, aunque no llega a tener una responsabilidad táctica como el otro especialista en voleibol; el colocador, debe ser un excelente observador del juego del equipo contrario, es decir debe interpretar continuamente las acciones tácticas y estratégicas del equipo rival. Esta exigencia táctica le permitirá un mejor posicionamiento en el campo, tanto de él mismo como de sus compañeros y así poder ayudar en la correcta posición de recepción y defensa de sus compañeros. La preparación táctica específica del líbero es fundamental, sobre todo en la faceta de recepción del saque, ya que tiene que trasladar a la recepción el planteamiento táctico de ataque del equipo, y adaptarla a las variaciones que pueden ocurrir en los diferentes momentos y fases el partido.

El jugador líbero debe poseer una correcta visión de campo y trabajar intensamente el juego de espaldas a la red (protección de tercer toque en jugadas falladas). Habitualmente, el jugador actúa sustituyendo a uno de los jugadores, normalmente los centrales, en su fase de defensa de campo, por ello, debe tener un adecuado concepto táctico que le permita defender en las zonas que son más exigidas. La atención y la memoria táctica de equipo son tareas básicas de las funciones del líbero.

Peculiaridades psicológicas de las tareas del líbero. Como se ha comentado anteriormente, para abordar la adecuada preparación psicológica de los especialistas debemos recordar y reconocer, clara y muy específicamente, las peculiaridades de sus funciones y tareas en la dinámica del equipo. Conocer con claridad las tareas técnico tácticas de los especialistas es la primera, y más importante labor para poder realizar un adecuado entrenamiento psicológico. 
Estas condiciones técnico-tácticas requieren de los especialistas unas particularidades psicológicas y mentales diferentes a las del resto de jugadores, que debe trabajar y entrenar. En algunas modalidades, como la que nos ocupa, esas condiciones resultan muy claras, relevantes y exclusivas y deben recibir una especializada preparación psicológica. Cada profesional que tenga que trabajar con los especialistas debe conocer cuáles son sus tareas tácticas más relevantes y exclusivas. En el caso de los líberos destacaremos como tareas tácticas exclusivas las siguientes:

Constantes cambios. Sustituye a determinados jugadores y siempre en momentos concretos, aunque secuenciados, por lo tanto, debe estar continuamente entrando y saliendo a la cancha e interrumpir momentáneamente su actividad física, técnica, táctica y mental. En algunas ocasiones la salida de la cancha puede ocurrir sin haber tenido la posibilidad de participar en el juego. Estas particularidades exclusivas de su función, requieren una preparación psicológica enfocada a lograr un alto nivel competitivo que le permita jugar en situaciones adversas y una continua adaptación mental a situaciones diferentes y cambiantes.

Participación interrumpida. Este especialista pasa de una actividad muy intensa a una actividad de pausa y, en pocos segundos, a un nuevo periodo de actividad. Esta característica de su juego, le ocasiona continuos cambios y adaptaciones en su nivel de activación y alteraciones en su capacidad atencional. Esta situación táctica de cambios e interrupciones en su actividad implica que el líbero debe realizar continuas adaptaciones y refocalizaciones atencionales.

Información táctica. También tiene que aplicar y comunicar las novedades estratégicas, que en forma de información, el entrenador quiera trasmitir a los jugadores en cancha. Es el auténtico vehículo transmisor de información del entrenador a los jugadores La información es variable y cambiante, y el líbero, por tanto, debe conocer perfectamente la táctica y poseer una buena capacidad de retención para trasmitirla a los compañeros en cancha. Por tanto, la atención y la memoria táctica son dos habilidades psicológicas básicas que debe desarrollar el líbero para desempeñar bien las exigencias mentales de su juego.

Las peculiaridades del puesto de especialista en todas sus dimensiones precisan que éstos tengan que desarrollar y entrenar habilidades psicológicas específicas, como se puede observar en el trabajo con los líberos, con los que se debe trabajar la memoria a corto plazo, así como capacidad de comunicación verbal rápida y altas capacidades de autocontrol. Estas habilidades requieren entrenamientos psicológicos específicos.

Habilidades psicológicas para las tareas del especialista líbero. Como hemos comentado repetidas veces, la preparación psicológica de los especialistas depende de las particularidades de las funciones tácticas que tiene encomendadas. Por lo tanto, se deben entrenar todas habilidades requeridas para conseguir una continúa readaptación mental a las cambiantes situaciones que permitan un alto nivel competitivo.

Ya se ha comentado que los continuos cambios produce que la salida y entrada de la cancha puede ocurrir sin haber tenido la posibilidad de participar en el juego, tal y como sucede cuando el equipo contrario comete un error en el saque, en cualquier caso, tiene que pasar de una actividad muy intensa a una actividad de pausa $y$, en pocos segundos, a un nuevo 
periodo de actividad. Esta característica de su juego, le ocasiona continuos cambios y regulaciones en su nivel de activación y modificaciones de sus condiciones atencionales

También, la información táctica de comunicar las novedades estratégicas, que el entrenador quiere trasmitir a los jugadores en cancha, supone que el líbero debe mejorar las habilidades de atención y la memoria táctica.

Así mismo, la actividad receptora requiere altas capacidades de concentración y una maleabilidad de cambios de orientación hacia los diferentes focos atencionales.

Otra de las tareas estratégicas fundamentales del líbero consiste en advertir constantemente a sus compañeros sobre sus posiciones, tanto en la fase de recepción del saque como en la fase defensiva. También debe advertir a sus compañeros sobre la necesidad de recuperar las posiciones defensivas una vez finalizada la correspondiente fase de juego.

Todas estas actividades tácticas propias de recepción y defensa, además de las que son exclusivas precisan que el líbero tenga habilidades psicológicas de memoria a corto plazo, así como desarrollar altas capacidades de comunicación verbal y no verbal y buena capacidades de autocontrol.

El entrenamiento psicológico del libero.Como se puede apreciar, la carga psicológica de la actividad y de las tareas de los líberos es muy exigente. Se requieren, por tanto, entrenamientos psicológicos específicos, tanto individualizados como integrados, para la instauración y optimización de las habilidades psicológicas que se necesitan en el perfeccionamiento de la actividad de los especialistas durante los partidos.

Para el entrenamiento psicológico del líbero debemos poner énfasis en las habilidades psicológicas que hemos venido exponiendo y, especialmente, en las que consideramos más trascendentes:

- Atención y concentración.

- Fortaleza mental

- Motivación intrinseca.

- Capacidad de liderazgo.

- Comunicación.

- Autoconfianza

- Control emocional

Por regla general son habilidades psicológicas comunes a otros especialistas, que deben ser entrenadas de formas diferentes y con distintas técnicas psicológicas. En el trabajo sobre los líberos hemos utilizado algunas técnicas concretas para entrenar las distintas habilidades psicológicas requeridas.

\section{Técnicas de entrenamiento psicológico.}

Entrenamiento para la atención-concentración. El primer objetivo del entrenamiento psicológico para los especialistas en recepción es ser capaces de alternar y cambiar de foco atencional y utilizarlos en los momentos adecuados. 
En un primer momento el líbero deberá tener claros los objetivos técnico- tácticos de su recepción. Estas tareas se centran fundamentalmente en controlar el balón y situarlo en las manos o zona del colocador para construir el ataque.. Para este cometido le va resultar muy útil el manejo de los diferentes focos atencionales anteriormente analizados. El líbero ha de ser consciente de dar tanta o más importancia a las fases previas al contacto con el balón, que al propio contacto.

Justo en el momento antes del saque el líbero deberá proponerse como prioritario centrarse en la focalización reducida-interna como ya se ha destacado, es decir en vigilar la situación técnica: posición de extremidades, brazos, manos, antebrazos, flexión de piernas, centro de gravedad, espalda, rigidez de brazos y antebrazo, etc.

La habilidad de focalización reducida-interna le ayudará, también, a conseguir centrase en sus pensamientos, sus emociones, sus seguridades. Este entrenamiento psicológico le permitirá, por tanto, poner las bases adecuadas para la consecución del objetivo técnicotáctico: controlar y precisar el balón.

La permanencia en este foco atencional será la del tiempo que dure la realización del saque, aproximadamente entre 3-8 segundos. La focalización apropiada será el fundamento para atender a lo que es realmente importante en este preciso momento, sin distracciones. Con este entrenamiento el líbero conseguirá una mayor motivación intrínseca, esto es, la motivación por la mejora personal. La motivación intrínseca aumentará los esfuerzos del jugador por mejorar y, además, le ayudará a que ésta no dependa únicamente de situaciones o estímulos externos como los errores, aciertos, elogios, aplausos, reproches..., es decir; de la motivación extrínseca.

Otro de los efectos positivos de la utilización adecuada de una focalización interna-reducida en este momento de espera, que implica una participación activa de intensidad menor, como es la recepción (Díaz y Díaz, 2012) es la evitación de estímulos distractores o de información irrelevante o disruptiva.

En el segundo momento del proceso de la recepción, el objetivo que debe conseguir el líbero, al tener que pasar a una fase de participación de actividad intensa, es el de cambiar su foco atencional y saltar a un estilo externo-amplio. El jugador, en esta fase, deberá dejar de prestar atención hacia dentro, para hacerlo ahora hacia fuera, en una dimensión atencional externa-amplia dirigida al jugador que saca, al tipo de saque, a la trayectoria del balón, a calcular la potencia del saque, sus efectos, etc. A medida que el balón llega a su zona, de nuevo, el receptor debe cerrar su foco y pasar a una dimensión externa-reducida. Ahora, su atención se debe centrar en el balón en el momento de ser controlado, en su contacto con los antebrazos, o con las palmas de las manos, o los dedos y darle una dirección adecuada para conseguir la precisión. Una vez que finaliza esta fase de recepción, de control y precisión del balón, el líbero debe pasar inmediatamente a poner en funcionamiento otra dimensión o estilo atencional para realizar la tarea de defensa.

Rutinas de preactuación. El empleo de rutinas se ha revelado como una práctica útil a la hora de establecer pautas de comportamiento óptimas tanto en los entrenamientos como en las competiciones. Las rutinas de preactuación servirán también para prevenir las pérdidas 
de concentración que se producen por el "decremento del calentamiento", que no es otra cosa que la disminución temporal de la eficacia con la que se realizan las tareas y actividades posteriores a un breve periodo de descanso, hasta que el jugador vuelva a estar completamente metido en la tarea (González, 2006)

Las frecuentes y cortas interrupciones en la actividad de los líberos suponen que continuamente deban hacer algún tipo de rutinas de preactuación para conseguir la disposición mental adecuada. Hay tantos tipos de rutinas previas a la vuelta a la actividad como jugadores. Sin embargo, las rutinas son más recomendables para las acciones cerradas. En voleibol, por ejemplo, el saque y en menor medida la recepción, son acciones o tareas cerradas. Los tiros libres en baloncesto.

Todas estas actuaciones ayudarán a los jugadores a mantener la concentración durante los momentos de actuación. Es conveniente que el jugador practique estas rutinas en las sesiones de entrenamiento.

Las rutinas deben, como hemos recogido en las observadas a los líberos, combinar actividades fisiológicas, comportamentales y psicológicas para estabilizar el comportamiento emocional y concretar adecuadamente el foco atencional a los estímulos relevantes.

Práctica imaginada. El ensayo mental o práctica imaginada es otra de las técnicas empleadas para el entrenamiento en focalización y concentración. Práctica imaginada, visualización o ensayo mental son algunas de las denominaciones que se refieren en el mundo del deporte a la recreación o vivencia mental de un hecho o tarea deportiva. Se trata de que el especialista viva mentalmente, mediante imágenes, la serie de situaciones que debe realizar durante su actuación. La experiencia y el conocimiento táctico del jugador son fundamentales para la correcta realización de esta técnica. El jugador realizará esta vivencia imaginada de futuro apoyada en su experiencia de pasado y sus conocimientos de los equipos y adversarios.

La práctica imaginada es una técnica que se debe adaptar a cada una de las fases que queremos entrenar mentalmente. Podemos repetirla todas las veces que consideremos necesario hasta lograr la automatización del gesto entrenado. Cada profesional deberá ir adaptado la práctica a cada una de las facetas que quiera entrenar o preparar. Como hemos dicho, quizás el entrenamiento de la concentración con práctica imaginada es más fácil en acciones cerradas, como el saque o la recepción, los penaltis, los tiros libres. Otras acciones abiertas requieren un trabajo de preparación de los ejercicios de práctica imaginada más detallistas.

El conocimiento o el visionado de videos de la variabilidad y sistemas de jugadores y equipos adversarios ayudarán mucho al trabajo con esta técnica. También la estadística nos aportará las probabilidades de en qué rotaciones los equipos utilizarán determinados tipos de saque y con qué trayectorias, a su vez nos facilitará el trabajo de practica imaginada y posibilitará que las imágenes mentales se acerquen con exactitud a la realidad.

Por lo tanto la estadística y los videos son herramientas complementarias y muy útiles para el trabajo psicológico de los especialistas.

Auto verbalizaciones instruccionales. Otra de las técnicas utilizadas para el entrenamiento psicológico son las autoverbalizaciones instruccionales. Para el enfrentamiento imaginado 
o mental de una determinada situación, y para poder conseguir la adecuada focalización a los estímulos y señales más influyentes en la actividad deportiva, el jugador podrá ayudarse de verbalizaciones internas para darse las instrucciones adecuadas. Practica imaginada y autoverbalizaciones pueden utilizarse conjuntamente y de forma complementaria.

De la misma forma, las autoverbalizaciones instruccionales servirán de guía para que el jugador se concentre en cada momento en las acciones adecuadas que debe realizar durante la competición real. Son verbalizaciones internas apropiadas que permiten afrontar la situación técnico-táctica de forma adecuada. Estas autoconversaciones internas ayudan a focalizar la atención en los estímulos y situaciones más relevantes. yudan a evitar aquellos que son irrelevantes y que incluso pueden descentrar o equivocar a los jugadores.

Cuanta más calidad y mayor relevancia tengan las preguntas que el jugador se haga, las respuestas serán más significativas y, por tanto, mayor eficiencia tendrá esta técnica.

Las autoinstrucciones en combinación con la práctica imaginada ayudaran a realizar las tareas de forma más correcta y permitirán actuar de forma automática. El jugador deberá enfrentarse mentalmente a las situaciones de forma positiva. Preguntarse y responderse internamente de forma adecuada. Es decir: hacerse la pregunta precisa y responderse con la respuesta más apropiada para resolver adecuadamente la situación táctica a la que se enfrenta.

Técnicas de entrenamiento psicológico para mejorar las habilidades de comunicación en los especialistas, la autoconfianza, el autocontrol, la motivación y el liderazgo.

Entrenamiento de la comunicación. Los especialistas, deben ser buenos comunicadores, buenos motivadores y grandes animadores del equipo. Su liderazgo defensivo y sus continuas entradas y salidas de la cancha, en el caso de los líberos, le permiten mantener un contacto directo con los jugadores que en ese momento juegan y los que esperan su oportunidad en el banquillo. La peculiaridad de este puesto les permite recibir continuamente órdenes de los técnicos para trasmitirlas a los jugadores en cancha. La entrada del líbero en cancha es, generalmente, recibida por los compañeros y por el público como una situación nueva y distinta. Es muy perceptible como su llegada es recibida con cierta animación en la cancha por su rapidez y por su conducta dinámica.

Dos tipos de comunicación deben ser conocidos y entrenados por los especialistas: verbal y no verbal

La comunicación Verbal. La necesidad de eficacia, brevedad, variedad y constancia de los mensajes, induce a que los especialistas deban emplear códigos comunes sencillos y conocidos por todos sus compañeros, de forma que una palabra única trasmita el contenido de un mensaje más amplio. Los líberos deben crear sus propios códigos, tanto verbales como no verbales. De hecho, todos los especialistas utilizan señales previamente pactadas.

La comunicación no verbal. Una parte significativa de la comunicación que se produce en el trascurso de un partido es de carácter no verbal. Las necesarias y continuadas entradas y salidas a la cancha de los líberos forman ya parte de esta comunicación. En general, los especialistas tienen que ser jugadores expresivos. Los entrenadores suelen pedirles que sean animosos, que mantengan el ambiente y la activación del equipo. 
Los líberos continuamente envían mensajes no verbales a sus compañeros. Los líberos deben cuidarse, si quieren cumplir sus funciones psicológicas, de ser positivos y animadores y no enviar mensajes negativos. Deben ser conscientes de la importancia de esta forma de comunicación. La entrada del líbero en la cancha se percibe por el equipo y por los aficionados como la ayuda de un experto. Es decir, de alguien que es bueno en las tareas propias.

La actividad comunicativa de los especialistas deberá mantener la moral competitiva de sus compañeros en un nivel óptimo, tanto cuando el marcador es favorable como, y fundamentalmente, cuando es adverso.

Aunque cada jugador debe esforzarse por mantener un estado de comunicación gestual adecuada, el líbero, como especialista, debe intentar influir en sus compañeros apoyando tanto las buenas acciones como los errores, para ayudarles a conseguir esa comunicación gestual positiva.

Deberán aprender a expresar sus emociones de forma positiva de tal forma que sus gestos, sus expresiones y su contacto físico con los compañeros manifiesten apoyo, aliento y refuerzo, de manera que entre ellos se establezca una relación de empatía, de ayuda mutua, de colaboración y de complicidad. Se debe impulsar una relación interpersonal optima en la que cada jugador aporta conductas y expresiones de interés auténtico por el juego del resto de compañeros, por mejorar su juego y para que éste ayude a conseguir una mayor eficacia individual y colectiva. Del mismo modo, comunicaciones inapropiadas pueden influir negativamente en el estado anímico del equipo. Si los mensajes y señales que emiten son inconvenientes e inoportunos el ambiente de equipo durante la competición no será el más adecuado

Entrenamiento de la autoconfianza. La autoconfianza es una habilidad fundamental para conseguir altos rendimientos deportivos. Las dos únicas acciones que realizan los líberos, la recepción y la defensa, son acciones que no proporcionan puntos al equipo. Son acciones en las que, además, es difícil, por lo general, conseguir buenas estadísticas.

Realizar una buena defensa ante un remate potente es singularmente difícil y, normalmente, suelen ser más las acciones fallidas que las acertadas. Por lo tanto, no gozan de tanta popularidad interna, es decir, que en muchas ocasiones no reciben una alta valoración del propio grupo, ni externa, es decir, la proveniente del público y espectadores, como sí ocurre con los rematadores, bloqueadores o sacadores.

Es cierto que en determinados momentos muy espectaculares y puntuales reciben refuerzos y apoyos positivos de compañeros y entrenadores que valoran su trabajo. Sin embargo, son muchos los entrenadores que opinan que es una labor poco reconocida por el público y los medios de comunicación y, seguramente, hasta poco comprendida en general. La seguridad y la convicción de que su aportación al equipo es trascendente serán la herramienta que ayudarán a mantener y mejorar la autoconfianza. Compañeros y entrenadores son agentes básicos para ayudar en mantener esa capacidad.

Entrenamiento del control emocional.(Autocontrol). Como ya se ha mencionado, los especialistas al participar más en el juego tienen muchas oportunidades para cometer errores 
individualizados y sus fallos están muy personalizados. La presión de la evaluación personal es mayor que en otras jugadas protagonizadas por el resto de jugadores del equipo. Esta singularidad del juego de los líberos puede afectar directamente a la pérdida del control necesario para jugar de forma adecuada. El autocontrol de las emociones negativas producidas por un error, entre otros matices, consiste en que el error no afecte a la forma de actuar en las siguientes jugadas. Esta situación es esencial, definitiva, para conseguir el equilibrio mental que se precisan los especialistas. Si el jugador piensa mal, se mantiene en su error, tiene dudas sobre sus capacidades y pierde confianza en su juego, puede aparecer la pérdida de control sobre su comportamiento deportivo. Cuando un jugador pierde el control sobre su mente, se descontrola su conducta y, por tanto, su juego se altera. Al tratarse de un especialista esta condición repercute sobre el juego de todo el equipo.

La autoconfianza y el autocontrol son destrezas psicológicas que se complementan. Ambas deben ser trabajadas intensamente por todos los jugadores pero, especialmente, por aquellos que tienen tareas imprescindibles, por los líderes efectivos del juego, por aquellos que tiene una mayor ascendencia y trascendencia en el juego y en el rendimiento del equipo.

Buceta (1998) define el autocontrol como la autorregulación del estado psicológico general y de las propias conductas. Es decir, que el autocontrol para el líbero consistirá en mantener sus destrezas psicológicas, tanto básicas como centrales, en estados óptimos de rendimiento y en realizar de forma eficaz sus habilidades y tareas técnico-tácticas, durante todo el partido y en cualquier circunstancia del juego. Un ejemplo de trabajo de autocontrol lo podemos encontrar en unas recientes declaraciones del tenista Rafa Nadal que comentó "tras la ruptura [del saque a su favor] pude calmarme, abrir mi mente y pensar en lo que tenía que hacer".

Es decir, el autocontrol se convierte en el nexo de unión, en la argamasa que mantiene la asociación eficiente entre las habilidades psicológicas básicas (atención, concentración, comunicaciones, anticipación, activación...) y las correctas tareas y habilidades técnicotácticas y estratégicas. El autocontrol y la regulación psíquica le ayudará a tomar decisiones eficientes y en todo momento.

Los momentos de los partido son cambiantes: jugar con el marcador en contra, a favor, periodos de pausa, periodos de actividad intensa, periodos activos de intensidad menor, periodos de espera, momentos intensos o igualados, final de partido de tanteo ajustado, sets de tie-break, presión de la grada, decisiones arbitrales. Si a todo esto añadimos la actividad continuada de los especialistas, además de su vulnerabilidad a la mayor probabilidad de cometer errores, nos encontramos ante un jugador que tiene muchas oportunidades de descontrolarse.

La falta de control implica la pérdida de concentración, el desajuste del nivel de activación, la aparición de pensamientos negativos, la utilización de comunicaciones incorrectas, la pérdida de confianza y la toma de decisiones equivocadas. Cuando ocurre esto decimos que el jugador ha perdido el control. La trascendencia de las tareas del especialista obliga a que el autocontrol sea una habilidad central del entrenamiento psicológico para evitar que el juego se descomponga, se altere y que esta situación se trasmita al resto del equipo y, por tanto, al juego colectivo. El líbero, por tanto, tiene que ser capaz en todo momento 
y en todas las fases del juego de tener su mente y, por tanto, su juego bajo control. Debe controlar la ansiedad por el triunfo, la frustración que produce un error o fallo importante, el infortunio por la pérdida de un set o los pensamientos intrusivos que pueden aparecer en cualquier momento del partido, aunque generalmente durante los periodos de pausa o espera. En definitiva, debe potenciar el trabajo de autocontrol.

Liderar. Un gran número de técnicos mantienen que el líbero, que los especialistas deben ser los lideres efectivos y afectivos del equipo. Ya hemos comentado que se les solicita que sean motivadores y generadores de ambientes de juego positivos. En ocasiones, y como dice Carniegie (1996), "la manera de dar vale más de lo que se da". La forma de entrar en la cancha, los contactos físicos con los compañeros, la actitud alegre y positiva de actuar son señales y gestos de apoyo al grupo y muestran vitalidad y dinamismo, condiciones que siempre se relacionan con el buen ambiente grupal. Los especialistas deben entrenar estas formas de actuar para liderar la mentalidad del grupo.

Si pretende fortalecer el juego de los demás compañeros, los especialistas deben permanecer alegres, contentos y activos. Deben incorporar a sus conductas deportivas gestos y maneras que levanten la moral de los demás en los momentos que estos precisen fortalecer su confianza ante una situación adversa, por ejemplo, ante marcadores desfavorables. En definitiva, tienen que disfrutar con su juego y ser mantenedor del ambiente de equipo.

En otras ocasiones, deberán tratar de controlar la excesiva euforia, en otras la relajación, en otras intervenir en posibles discusiones o desacuerdos... En esto consiste la tarea psicológica que en muchas ocasiones los entrenadores piden a los especialistas. En resumen: que lideren.

Deben estar atentos a esas situaciones y empatizar con sus compañeros, es decir, ponerse en lugar emocional del resto del equipo. ¿Qué están sintiendo? ¿Qué les está pasando? ¿Cuáles son las preocupaciones que tienen? E intentar influir sobre ellos. Liderar el juego requiere la actitud y la predisposición competente, de manera que posibilite motivación y confianza a todo el grupo.

\section{Discusión y Conclusiones}

A lo largo del trabajo hemos repasado la trascendencia de las tareas que realiza el líbero como especialistas en el voleibol. También, hemos considerado que la importancia de esas tareas técnico-táctica deben ser soportadas en una adecuada e imprescindible preparación mental. El líbero, y los especialistas en general, deben, por tanto, entrenar y potenciar todas sus habilidades psicológicas. En general, mantenemos, con otros expertos en entrenamiento deportivo, que todos los especialistas deben y pueden contribuir a mejorar el rendimiento mental de los compañeros si actúan psicológicamente orientados. Los líberos, como todos los especialistas, necesitan, por tanto, a juicio de muchos entrenadores, una correcta automotivación, que debe ser fundamentada en una gran confianza en sus habilidades físicas y técnicas, en sus capacidades tácticas y en sus propias convicciones psicológicas. Los entrenamientos para mejorar sus capacidades de liderazgo, comunicación verbal y no verbal, el autocontrol emocional, con las técnicas psicológicas integradas son imprescindibles en el trabajo con los especialistas. Los especialistas han de tener conciencia de que domina todas 
las situaciones psicológicas del juego, tanto en el plano individual como en el colectivo. Los psicólogos del deporte debemos, en la línea que mantenemos en este trabajo, proponerles herramientas y técnicas psicológicas que les permitan mejorar sus destrezas comunicativas, sus capacidades de automotivación y motivación, sus capacidades de control y desarrollar habilidades de liderazgo que posibiliten la confianza de sus compañeros. Si los líberos, los especialistas, no desarrollan adecuadamente sus habilidades psicológicas, es decir, si no despliegan las adecuadas estrategias mentales para controlar y actuar correctamente en todas las situaciones del juego que, como hemos venido manteniendo, determinan y exigen una forma de actuar diferente y, en cambio, se deja arrastrar por los errores, las presiones o una activación excesiva, es inevitable que el rendimiento del grupo se vea alterado considerablemente.

En resumen, el presente trabajo propone que el trabajo psicológico con los especialistas con programas adecuados, con las técnicas precisas e integrados en el trabajo diario en la sesiones, es una magnifica forma de ensamblar el trabajo interdisciplinar de entrenadores y psicólogos del deporte. Creemos que el modelo propuesto para el trabajo psicológico con el líbero de voleibol es aplicable a otros especialistas con las particularidades propias de cada especialista. Sería conveniente que en próximos trabajos se abordaran y propusieran formas de actuación concreta con especialistas de otras modalidades deportivas y evaluar las ventajas competitivas de esas intervenciones.

\section{BIBLIOGRAFIA}

Antúnez, A., Ureña, F., Velandrino, A. P. y García, M. D. M. (2004). Valoración de la efectividad de interceptación con éxito delaportera debalonmanoanteellanzamientotrasla aplicación deun programa perceptivomotor. Rev. Int. Med. Cienc. act. fis. deporte, 15

Blanco, I. y Buceta, J. (1999). Intervención psicológica con el equipo nacional olímpico de hockey hierba femenino. $R e$ vista de Psicología del Deporte, 2 (1), 87 - 100

Bossio, M. R., Raimundi, M. J., y Correa, L. G. (2012). Programa de Entrenamiento en Habilidades Psicológicas en jugadoras de voleibol de alto rendimiento. Cuadernos de psicología del deporte, 12(1).

Buceta, J.M. (1992). Intervención psicológica con el equipo nacional olímpico de baloncesto femenino. Revista de Psicología del Deporte 1(2), 69 - 87.

Buceta, J.M. (1998) Psicología del entrenamiento deportivo. Madrid. Dykinson.

Carniegie,D. (1996) Como ganar amigos e influir sobre las personas. Madrid. Elipse.

Cruz, J. (1992). El asesoramiento y la intervención psicológica en deportistas Olímpicos. Revista de Psicología del Deporte 1 (2), 41 - 46.

De la Vega Marcos, R., Barquín, R. R., y Del Valle, S. (2010). Tendencia de acción de porteros de fútbol profesional: el caso de los penaltis. Cuadernos de psicología del deporte, 10(2), 23-32.

Díaz, J (2010) El entrenamiento mental de los jugadores de bolos. Preparación para el alto rendimiento. Santander. F.C.B

Díaz, J. y Díaz, I (2012) ¿Cuándo deben intervenir los entrenadores en la dirección de un partido de voleibol?: posibles intervenciones psicológicas durante las distintas fases competitivas de un partido. Cuadernos de Psicología 
del deporte Volumen 12. Núm.1 121-135

Escudero López, J. (1992) Preparación psicológica de la selección olímpica española de ciclismo: Barcelona 92. Revista de Psicología del Deporte, 1(2), 95 - 101

Eraña de Castro, I. (2004) Entrenamiento psicológico con jóvenes tenistas. Revista de Psicología del Deporte, 13 (2), 263 - 271.

González, A.M. (2006)Técnicas psicológicas de preferente aplicación en los bolos, la psicología del deporte de los bolos. Actas de las jornadas de Psicología de los bolos. FCB. Santander 37-54

González, J. y Garcés de los Fayos, E. (2009). Plan de entrenamiento psicológico en el deporte de la petanca: en búsqueda del rendimiento grupal óptimo. Revista de Psicología del Deporte, 18 (1), 87 - 104.

Iturriaga, F. M. A., Marcos, R. D. L. V.,y Barquín, R. R. (2015). Percepción de éxito y rendimiento deportivo de un portero de waterpolo/Perception of Success and Athletic Performance of a Water Polo Goalkeeper. Apunts. Educació física i esports, (122), 21.

Jiménez, A. C., Sáenz-López, P., Ibáñez, S. J. y Lorenzo, A. (2012). Percepción de las jugadoras internacionales de baloncesto sobre su toma de decisiones. Revista Internacional de Medicina y Ciencias de la Actividad Física y del Deporte, 12(47), 589-609

Mahl, A. y Vascocelos, J. (2007) Perfil psicológico de prestaçao de jogadores profissionais de futebol do Brasil. Revista Portuguesa de Ciencias do Desport, 7.1 80-91

Martínez, J. G., Bonet, A. C. y Encinas, F. L. (1998). Programa psicológico para mejorar los resultados de jugadores de balonmano. Psicothema, 10(2), 271-280.

Medina, A., Parra, M. D. M. G., Iturriaga, F. M. A., Lara, E. R. y Estero, J. L. A. (2010). Estudio piloto del efecto de un programa de entrenamiento perceptivo-motor sobre la eficacia en competición de la portera de balonmano según la situación del lanzador. Retos. Nuevas tendencias en Educación Física, Deporte y Recreación, (18), 29-34.

Nieto, G.y Olmedilla, A. (2001). Planificación del entrenamiento psicológico en atletas de élite: un caso en marcha atlética. Revista de Psicología del Deporte, 10 (1), 127 - 142

Noce, F., y Samulski, D. M. (2002). Análisis del estrés psíquico en colocadores brasileños de voleibol de alto nivel. Revista de Psicología del Deporte, 11(1), 0137-155.

Olmedilla, A., Ortega, E., Ortín, F. J. y Andreu, M. D. (2010). Programa de intervención psicológica en futbolistas. Revista de Psicología del Deporte, 19(2), 0249-262.

Pérez, G.; Marí, J. y Font, J. (1993). CAR: Entrenamiento psicológico para los Juegos Olímpicos Barcelona 92. Revista de Psicología del Deporte, 2 (1), 68 - 79.

Pulgarín,M.(1996).Diseñodeun programadeentrenamientopsicológicoenunequipofemeninodevoleibol.Revista de Psicología del Deporte, 5 (2), 67 - 76.

Sá, P., Gomes, A. R., Saavedra, M. y Fernández, J. J. (2015). Percepción de los porteros expertos en balonmano de los factores determinantes para el éxito deportivo. Revista de psicología del deporte, 24(1), 21-27.

Sánchez-Alarcos y Díaz-Pintado, J. V. (2015). Preíndices que determinan la anticipación del portero de hockey hierba ante un golpeo de revés. Tesis Doctoral .Departamento d’Educacio Física y Deportiva. Universitat de Valencia 